\title{
Study of collective radial breathing-like modes in double-walled carbon nanotubes: combination of continuous two-dimensional membrane theory and Raman spectroscopy
}

\author{
Dmitry I. Levshov, ${ }^{\text {a,* }}$ Marina V. Avramenko, ${ }^{\text {a }}$ Xuan-Tinh Than,,c \\ Thierry Michel, ${ }^{\mathrm{b}, \mathrm{c}}$ Raul Arenal, ${ }^{\mathrm{d}, \mathrm{e}}$ Matthieu Paillet, ${ }^{\mathrm{b}, \mathrm{c}}$ \\ Dmitry V. Rybkovskiy, ${ }^{a}$ Alexander V. Osadchy, ${ }^{\text {f,g }}$ Sergei B. Rochal, \\ Yuri I. Yuzyuk, and Jean-Louis Sauvajol ${ }^{\text {b,c }}$ \\ ${ }^{a}$ Southern Federal University, Faculty of Physics, 5 Zorge Street, Rostov-on-Don 344090, Russia \\ ${ }^{b}$ Université Montpellier 2, Laboratoire Charles Coulomb, Montpellier F-34095, France \\ ${ }^{\mathrm{c}}$ CNRS, Laboratoire Charles Coulomb, Montpellier F-34095, France \\ ${ }^{\mathrm{d}}$ Universidad de Zaragoza, Laboratorio de Microscopias Avanzadas, Instituto de Nanociencia de \\ Aragon, Zaragoza E-50018, Spain \\ eARAID Foundation, Zaragoza E-50018, Spain \\ ${ }_{\mathrm{f}}^{\mathrm{f} u s s i a n}$ Academy of Sciences, A. M. Prokhorov General Physics Institute, 38 Vavilov Street, \\ Moscow 119991, Russia \\ ${ }^{\mathrm{g}}$ National Research Nuclear University MEPhI (Moscow Engineering Physics Institute), 31 \\ Kashirskoye Shosse, Moscow 115409, Russia
}

\begin{abstract}
Radial breathing modes (RBMs) are widely used for the atomic structure characterization and index assignment of single-walled carbon nanotubes (SWNTs) from resonant Raman spectroscopy. However, for double-walled carbon nanotubes (DWNTs), the use of conventional $\omega_{\mathrm{RBM}}(d)$ formulas is complicated due to the van der Waals interaction between the layers, which strongly affects the frequencies of radial modes and leads to new collective vibrations. This paper presents an alternative way to theoretically study the collective radial breathing-like modes (RBLMs) of DWNTs and to account for interlayer interaction, namely the continuous twodimensional membrane theory. We obtain an analytical $\omega_{\mathrm{RBLM}}\left(d_{o}, d_{i}\right)$ relation, being the equivalent of the conventional $\omega_{\mathrm{RBM}}(d)$ expressions, established for SWNTs. We compare our theoretical predictions with Raman data, measured on individual index-identified suspended DWNTs, and find a good agreement between experiment and theory. Moreover, we show that the interlayer coupling in individual DWNTs strongly depends on the interlayer distance, which is manifested in the frequency shifts of the RBLMs with respect to the RBMs of the individual inner and outer tubes. In terms of characterization, this means that the combination of Raman spectroscopy data and predictions of continuous membrane theory may give additional criteria for the index identification of DWNTs, namely the interlayer distance. (c) 2015 Society of Photo-Optical Instrumentation Engineers (SPIE) [DOI: 10.1117/1.JNP.10.103599]
\end{abstract}

Keywords: individual double-walled carbon nanotubes; radial breathing-like modes; interlayer coupling; Raman spectroscopy.

Paper 15076SS received Jul. 31, 2015; accepted for publication Aug. 26, 2015; published online Sep. 22, 2015.

\section{Introduction}

A double-walled carbon nanotube (DWNT) has two concentric carbon layers. This structure makes it an ideal system for studying the effects of interwall coupling on the physical properties of carbon nanotubes (CNTs). Compared with single-walled carbon nanotubes (SWNTs),

*Address all correspondence to: Dmitry I. Levshov, E-mail: dmitry.levshov@gmail.com

1934-2608/2015/\$25.00 (C) 2015 SPIE 
DWNTs have higher mechanical strength and thermal stability and also possess interesting electronic and optical properties. ${ }^{1,2}$ A DWNT can have one of the four possible electronic configurations with each wall being either semiconductor (S) or metallic (M): S@S, M@S, M@M, and S@M (inner-tube@outer-tube). Every configuration may have different electronic properties.

The Raman scattering is the main analytical tool to study intrinsic properties of DWNTs. Spectroscopic experiments on DWNTs have been initially performed on solution-based samples or in bundles. ${ }^{3-5}$ Recently, more evolved experiments using combinations of different complementary techniques (e.g., atomic force microscopy, electron diffraction (ED), and resonant Raman spectroscopy) were performed by our and other groups to measure Raman spectra of individual DWNTs and to study the effects of van der Waals interaction. ${ }^{6-11}$

In our recent work, combining high-resolution transmission electron microscopy (HRTEM), ED, and resonant Raman spectroscopy on individual free-standing DWNTs, ${ }^{11}$ we have shown that the strength of this "inner" tube-"outer" tube interaction, which is dependent on the interlayer distance in DWNTs, strongly affects the out-of-phase radial breathing-like mode (RBLM) frequencies. We thus proposed that the positions of the radial breathing-like modes (RBLMs) and the difference between experimental and theoretical calculations within atomistic valence force field model ${ }^{12}$ can be used as criteria to evaluate diameters of the inner and outer tubes in nonindex-identified DWNTs.

This paper presents an alternative theoretical way to study low-frequency collective modes of DWNTs based on the two-dimensional (2-D) continuous membrane theory. We show that this approach provides a new and straightforward way to quantitatively analyze RBLMs of coupled systems.

\section{Experimental Details}

The individual double-walled carbon nanotubes were synthesized by the catalytic chemical vapor deposition directly onto commercial TEM grids (with holes up to $3 \mu \mathrm{m}$ in diameter). ${ }^{13,14}$

To study individual single- and multiwalled carbon nanotubes, we developed an experimental procedure based on the combination of resonant Raman spectroscopy, ED, and HRTEM. This approach provides an unambiguous way to identify the chiral indices of CNTs. ${ }^{6,15,16}$ In this work, TEM, HRTEM, and electron diffraction patterns (EDPs) were obtained in an FEI Titan microscope operating at $80 \mathrm{kV}$ to reduce damages induced by electron irradiation. ${ }^{14} \mathrm{TEM}$ images and EDPs were recorded within an average 5-s acquisition times.

Resonant Raman scattering measurements were carried out using a Jobin Yvon T64000 spectrometer equipped with a liquid nitrogen-cooled, silicon charge-coupled device detector. The scattered light was collected through a microscope using a backscattering configuration. In all the measurements, both incident and scattered light polarizations are along the nanotube axis (// // polarized Raman spectrum). Incident excitations from $\mathrm{Ar}+$ and $\mathrm{Kr}+$ lasers, dye laser, and tunable Ti/sapphire laser were used. To avoid heating effects, the laser power impinging the sample was kept below $50 \mu \mathrm{W}$ with a $100 \times$ objective (numerical aperture of 0.95 ). The low-frequency part (RBM region) of Raman spectra was fitted following standard procedures with a set of two Lorentzians in an OriginPro package.

\section{Results}

The Raman spectra of 14 identified individual free-standing DWNTs have been recorded and analyzed in detail. Figure 1 shows the RBLM range of the Raman spectrum measured on one of these individual DWNTs, namely $(23,5) @(22,17)$, with the corresponding HRTEM image and EDP. Our individual DWNTs are ultralong, clean from amorphous carbon, and have no signature of the D-band (not shown) in Raman spectra. ${ }^{13,14}$ Only five of these 14 individual DWNTs had both RBLMs observed in the Raman spectra. All the structural parameters for these five tubes and the measured $\omega_{\text {RBLMs }}$ are listed in Table 1. In addition to our own data, we will use the results of Liu et al. ${ }^{8}$ for the evaluation of our theoretical approach.

Recently, we have described the low-frequency dynamics of DWNTs in the framework of the continuous 2-D membrane theory (for its detailed theoretical description, applicability, and 

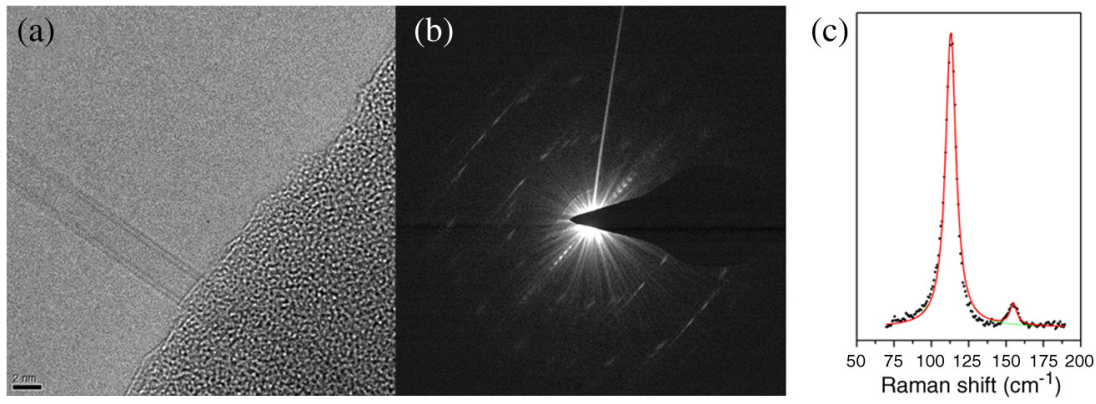

Fig. 1 (a) High-resolution transmission electron microscopy (HRTEM) image, (b) electron diffraction pattern, (c) and measured radial breathing-like modes (RBLMs) $(633 \mathrm{~nm})$ of the $(23,5) @$ $(22,17)$ double-walled carbon nanotube (DWNT) studied in this paper. Green lines correspond to the two individual Lorentzians, while red line stands for their superposition.

Table 1 The structural and vibrational information on the double-walled carbon nanotubes (DWNTs) studied in this work. The diameters $d$ are given in $\mathrm{nm}$ and frequencies $\omega$ in $\mathrm{cm}^{-1}$.

\begin{tabular}{lcccccc}
\hline \hline$\#$ & $(\mathrm{n}, \mathrm{m})$ indices & $d_{i}$ & $d_{o}$ & $\Delta d$ & $\omega_{L}^{\exp }$ & $\omega_{H}^{\exp }$ \\
\hline 1 & $(12,8) @(16,14)$ & 1.37 & 2.04 & 0.67 & 133 & 186 \\
2 & $(13,9) @(24,7)$ & 1.50 & 2.21 & 0.71 & 123 & 169 \\
3 & $(18,2) @(20,12)$ & 1.49 & 2.19 & 0.70 & 122 & 167 \\
4 & $(22,11) @(27,17)$ & 2.28 & 3.01 & 0.73 & 98 & 121 \\
5 & $(23,5) @(22,17)$ & 2.03 & 2.65 & 0.62 & 113 & 154 \\
\hline \hline
\end{tabular}

comparison with other models describing RBLMs, see Ref. 17). We treated each layer of DWNTs as a 2-D membrane composed of single atoms (or in other words having zero thickness) and not as a finite-thickness plate. Following this approach, we obtained the equation ${ }^{17}$

$$
\left[d_{i} \cdot\left(\omega_{i, \mathrm{SWNT}}^{2}-\omega^{2}\right)+2 G\right] \cdot\left[d_{o} \cdot\left(\omega_{o, \mathrm{SWNT}}^{2}-\omega^{2}\right)+2 G\right]-4 G^{2}=0,
$$

where $\omega$ are the RBLM frequencies of DWNT; $d_{o}$ and $d_{i}$ are the diameters of outer and inner layers respectively; $G=f\left(d_{o}, d_{i}\right)$ ( $\mathrm{G}$ unit is $\mathrm{cm}^{-2} \mathrm{~nm}$ when the frequencies are in $\mathrm{cm}^{-1}$ and the diameters in nm, see Ref. 16) is a coupling function between the two layers (see below for more information); and finally $\omega_{i, \mathrm{SWNT}}^{2}$ and $\omega_{o, \mathrm{SWNT}}^{2}$ are the RBM frequencies of the isolated individual inner and outer layers.

We now further develop the ideas of the above-mentioned work. By simplifying Eq. (1), we obtain the formula

$$
\omega_{\mathrm{RBLM}}=\sqrt{\frac{b \pm \sqrt{b^{2}-4 c}}{2}},
$$

with parameters $b, c$ being defined as

$$
\begin{gathered}
b=\omega_{o, \mathrm{SWNT}}^{2}+\omega_{i, \mathrm{SWNT}}^{2}+\frac{2 G \cdot\left(d_{o}+d_{i}\right)}{d_{o} \cdot d_{i}} \\
c=2 G \cdot\left(\frac{\omega_{o, \mathrm{SWNT}}^{2}}{d_{i}}+\frac{\omega_{i, \mathrm{SWNT}}^{2}}{d_{o}}\right)+\left(\omega_{o, \mathrm{SWNT}} \cdot \omega_{i, \mathrm{SWNT}}\right)^{2},
\end{gathered}
$$

and 


$$
\omega_{i(o), \mathrm{SWNT}}=\frac{227}{d_{i(o)}} \sqrt{1+C_{e} \cdot d_{i(o)}^{2}} \text {. }
$$

Equation (2) is an equivalent of well-known $\omega(d)$ relations established for SWNTs. ${ }^{18}$ It provides a powerful and easy-to-use tool to analyze coupled radial breathing-like vibrations as it considers (a) all different interlayers distances $\Delta d$ and (b) different environment conditions (constant $\left.C_{e}\right)$. For every DWNT, defined by a couple of diameters $\left(d_{o}, d_{i}\right)$, Eq. (2) gives two frequencies corresponding to the in-phase RBLM $\left[\omega_{L}\right.$, sign "--" in Eq. (2)] and the out-of-phase RBLM $\left[\omega_{H}\right.$, sign "+" in the Eq. (2)]. The constant $C_{e}$ in Eq. (5) describes different environments for the CNTs. ${ }^{18}$

It is also clear from Eqs. (2)-(4) that the knowledge of the coupling function $G=f\left(d_{o}, d_{i}\right)$ is necessary for the calculations of $\omega_{\text {RBLM }}$. In the absence of the exact theoretical form, it is possible to determine it from the fit of experimental data. For instance, the empirical form of the coupling function $G=f\left(d_{o}, d_{i}\right)$ was previously determined ${ }^{17}$ on the basis of experimental data of Liu et al., ${ }^{8}$ for which the absence of external interaction $\left(C_{e}=0\right)$ on both tubes was stated. It is expressed as $G=\left[A+B\left(d_{o}-d_{i}\right)+C\left(d_{o}+d_{i}\right)\right]\left(d_{o}+d_{i}\right)$ with $A=7210 \mathrm{~cm}^{-2}$, $B=-9670 \mathrm{~cm}^{-2} / \mathrm{nm}$, and $C=61 \mathrm{~cm}^{-2} / \mathrm{nm}$. ${ }^{16}$ The normalized intertubes coupling, $G_{\text {norm }}$ (equivalent to unit-area force constant of Ref. 8), defined as $G /\langle d\rangle$ with $\langle d\rangle=\left(d_{o}+d_{i}\right) / 2$, provides a unique way to evaluate how the van der Waals interactions vary with $\Delta d$. For the Liu et al. data, this function is plotted in Fig. 2 (black circles).

We now calculate the $G_{\text {norm }}$ values for our individual DWNTs. It was previously found that in the framework of the atomistic model, RBLM frequencies of $(12,8) @(16,14)$ DWNT are best described, if $C_{e}=0.065$ for the outer layer and $C_{e}=0$ for the inner layer are considered. ${ }^{6,11}$ Because all DWNTs, studied in this work, were synthesized following the similar procedure, we use the same $C_{e}$ values. Under this assumption, we find that the calculated $G_{\text {norm }}$ values for our DWNTs (red rhombs in Fig. 2) wind around the $G_{\text {norm }}$ values derived from the fit of Ref. 8. This confirms that the intertubes coupling $G_{\text {norm }}$ mainly dependent on the interlayer distance $\Delta d$, independently of the structure and environment of the constituent outer and inner SWNTs.

Finally, we illustrate the practical use of our model on the example of the work of Liu et al., ${ }^{8}$ for which more experimental data are available and the $G$ function is better established. By choosing the proper form of $G$ (see above) and using the Eq. (2), we can calculate a set of $\omega(d)$ relations for different interlayer distances, $\Delta d=d_{o}-d_{i}$, both for in-phase $\omega_{L}$ and out-of-phase $\omega_{H}$ RBLMs. These calculated curves are shown in Fig. 3 as solid thin lines, with $\Delta d$ in the range 0.60 to $0.76 \mathrm{~nm}$ with a step of $0.02 \mathrm{~nm}$. In addition, the results of calculations are compared with the $\omega=228 / d$ relation (black-dotted dashed line) and $\omega_{\text {RBLMs }}(d)$ relation, obtained from the atomistic valence force field model with $\Delta d=0.68 \mathrm{~nm}$ and without adjustable parameters, (thick black-dashed line), which was used in our previous works. ${ }^{6,711,19}$

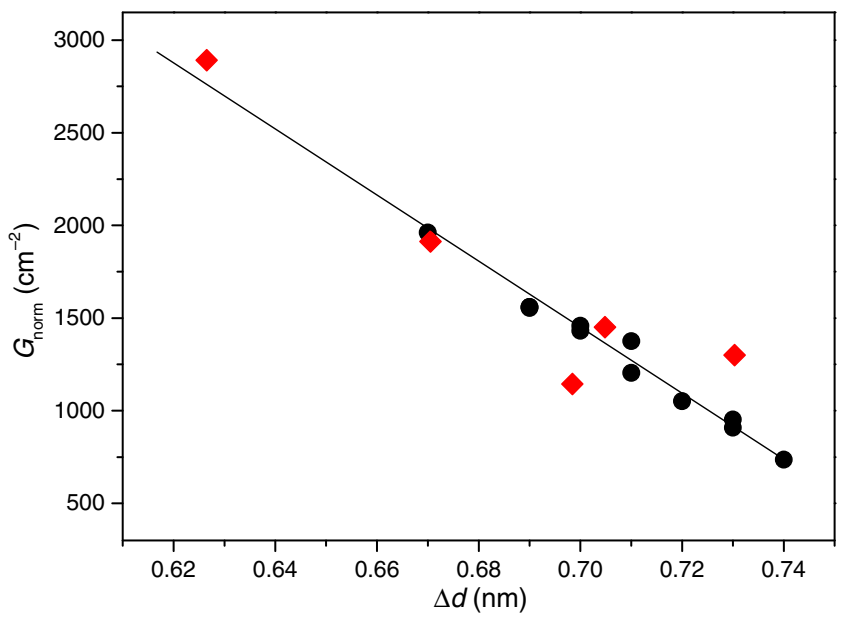

Fig. 2 The coupling $G_{\text {norm }}$ as a function of interlayer distance calculated within continuous membrane theory using the available experimental data from Ref. 8 (black circles) and from the present work (red rhombs). The line is a guide for eyes. 

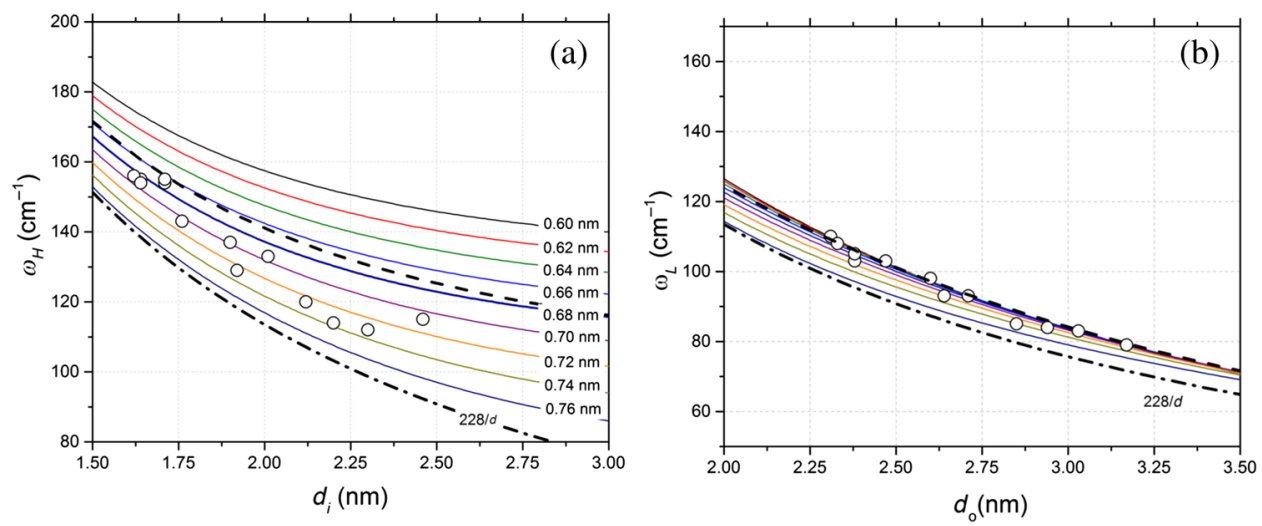

Fig. 3 Theoretical $\omega_{\text {RBLM }}(d)$ relations (shown as solid thin lines), calculated using Eq. (2) for (a) out-of-phase $\left(\omega_{H}\right)$ and (b) in-phase $\left(\omega_{L}\right)$ RBLMs for different interlayer distances (with $\Delta d$ in the range 0.60 to $0.76 \mathrm{~nm}$ with a step of $0.02 \mathrm{~nm}$ ), and compared with the $\omega=228 / d$ relation (black dotted dashed line) and $\omega_{\text {RBLMs }}(d)$ obtained from the atomistic valence force field model (thick black-dashed line). ${ }^{12}$ The experimental data of Liu et al. ${ }^{8}$ are represented by open circles.

The experimental data of Liu et al. ${ }^{8}$ are represented by open circles in Fig. 3. As expected, at large $\Delta d$ the $\omega_{\mathrm{RBLM}}(d)$ relations tend to $\omega=228 / d$, or in other words to the case of noninteracting layers. From the analysis of Fig. 3, we can clearly see that the out-of-phase mode, $\omega_{H}$, is much more affected by the change of the interlayer distance than the in-phase mode, $\omega_{L}$. Therefore, it is possible to estimate the interlayer distance of the given DWNT from the comparison between the relative position of the measured out-of-phase $\omega_{H}$ and the calculated $\omega_{\mathrm{RBLM}}(d)$ relations. In this way, the predictions of continuous membrane theory may give additional criteria for the index-identification of DWNTs.

\section{Conclusions}

We presented an alternative way to calculate low-frequency collective modes of DWNTs: 2-D continuous membrane theory. Following this approach, we have obtained a $\omega_{\mathrm{RBLM}}\left(d_{o}, d_{i}\right)$ relation being an equivalent to the conventional formulas established for individual SWNTs.

This approach was first applied to the understanding of the experimental data of Ref. 8 for which the absence of external interaction $\left(C_{e}=0\right)$ on both tubes was stated. A monotonic dependence of the normalized intertubes coupling, $G_{\text {norm }}$, with $\Delta d$ is clearly stated. A close dependence of $G_{\text {norm }}$ is found from the fit of our data meaning that the normalized intertubes coupling in DWNTs indeed depends on the interlayer distance $\Delta d$.

In terms of characterization, the combination of Raman spectroscopy data and the predictions of continuous membrane theory may give additional criteria for the index identification of DWNTs.

\section{Acknowledgments}

T. T. and D. L. performed synthesis of individual double-walled carbon nanotubes. D. L., T. M., M. P., and J.-L. S. devised and performed Raman experiments. D. L. and R. A. identified atomic structures of the nanotubes by electron diffraction and HRTEM. M. A. and S. R. devised 2-D membrane theory and made calculations of RBLM frequencies of individual DWNTs. D. L., M. A., D. R., A. O., T. M., R. A., M. P., J.-L. S., S. R., and Yu. Y. actively participated in the data analysis, discussions, and wrote the paper. D. L., M. A., D. R., and S. R. gratefully acknowledge the financial support of Russian Science Foundation (Grant No. 15-12-10004). R. A. gratefully acknowledges the support of the Spanish Ministerio de Economia $y$ Competitividad (FIS2013-46159-C3-3-P) and funding from the European Union Seventh Framework Program under Grant No. 312483-ESTEEM2 (Integrated Infrastructure InitiativeI3). The microscopy work was conducted in the Laboratorio de Microscopias Avanzadas at the Instituto de Nanociencia de Aragon, Universidad de Zaragoza (Spain). 


\section{References}

1. C. Shen, A. H. Brozena, and Y. Wang, "Double-walled carbon nanotubes: challenges and opportunities," Nanoscale 3(2), 503-518 (2011).

2. R. Pfeiffer et al., "Double-wall carbon nanotubes," Top. Appl. Phys. 111, 495-530 (2008).

3. H. Kuzmany et al., "Raman scattering from double-walled carbon nanotubes," J. Raman Spectrosc. 39(2), 134-140 (2008).

4. R. Pfeiffer et al., "Interaction between concentric tubes in DWCNTs," Eur. Phys. J. B 42(3), 345-350 (2004).

5. R. Pfeiffer et al., "Fine structure of the radial breathing mode of double-wall carbon nanotubes," Phys. Rev. B 72(16), 161404 (2005).

6. D. Levshov et al., "Experimental evidence of a mechanical coupling between layers in an individual double-walled carbon nanotube," Nano Lett. 11(11), 4800-4804 (2011).

7. D. Levshov et al., "Comparative Raman study of individual double-walled carbon nanotubes and single-walled carbon nanotubes," J. Nanoelectron. Optoelectron. 8(1), 9-15 (2013).

8. K. Liu et al., "Quantum-coupled radial-breathing oscillations in double-walled carbon nanotubes," Nat. Commun. 4, 1375 (2013).

9. T. C. Hirschmann et al., "Characterization of bundled and individual triple-walled carbon nanotubes by resonant Raman spectroscopy," ACS Nano 7(3), 2381-2387 (2013).

10. T. C. Hirschmann et al., "Role of intertube interactions in double- and triple-walled carbon nanotubes," ACS Nano 8(2), 1330-1341 (2014).

11. D. Levshov et al., "Coupled vibrations in index-identified carbon nanotubes," MRS Proc. 1700, 69-77 (2014).

12. V. Popov and L. Henrard, "Breathinglike phonon modes of multiwalled carbon nanotubes," Phys. Rev. B 65(23), 235415 (2002).

13. T. X. Tinh et al., "Synthesis of individual ultra-long carbon nanotubes and transfer to other substrates," J. Exp. Nanosci. 6(5), 547-556 (2011).

14. R. Arenal et al., "Direct evidence of atomic structure conservation along ultra-long carbon nanotubes," J. Phys. Chem. C 116(26), 14103-14107 (2012).

15. J. Meyer et al., "Raman modes of index-identified freestanding single-walled carbon nanotubes," Phys. Rev. Lett. 95(21), 217401 (2005).

16. T. Michel et al., "About the indexing of the structure of single-walled carbon nanotubes from resonant Raman scattering," Adv. Nat. Sci. Nanosci. Nanotechnol. 1(4), 045007 (2010).

17. S. B. Rochal, V. L. Lorman, and Y. I. Yuzyuk, "Two-dimensional elasticity determines the low-frequency dynamics of single- and double-walled carbon nanotubes," Phys. Rev. B 88(23), 235435 (2013).

18. P. Araujo et al., "Nature of the constant factor in the relation between radial breathing mode frequency and tube diameter for single-wall carbon nanotubes," Phys. Rev. B 77(24), 241403 (2008).

19. R. Parret et al., "Raman spectroscopy on individual identified carbon nanotubes," MRS Proc. 1407 (2012).

Dmitry I. Levshov received his MSc degree in physics from the Southern Federal University, Rostov-on-Don, Russia, in 2010 and his PhDs in physics from the University Montpellier 2, Montpellier, France, and Southern Federal University, Rostov-on-Don, Russia, in 2013. His research interests include carbon nanotube growth, study, and characterization. In particular, he focuses on the study of intrinsic properties of individual carbon nanotubes by combination of Raman spectroscopy, electron diffraction, and high-resolution electron microscopy.

Marina V. Avramenko received her MS degree in nanotechnology and microsystems engineering from the Southern Federal University, Rostov-on-Don, Russia, in 2014. Currently, she is in the second year of the physics and astronomy PhD program at Southern Federal University in professor Rochal's group. Her research interests include theoretical investigation of vibrational and thermal properties of carbon nanotubes. 
Xuan-Tinh Than received his BSc degree in organic chemistry in 2005 and his MSc degree in engineering physics and nanotechnology from Vietnam National University, Hanoi, Vietnam, in 2008 and his PhD in physics from the University Montpellier 2, Montpellier, France, in 2008. His research interests include synthesis of carbon nanotubes, nitrogen-doped carbon nanotubes, Raman spectroscopy of nanotubes, and application of nanotubes as a catalyst in electrodes of fuel cells.

Thierry Michel received his MS degree in materials science in 2003 and his $\mathrm{PhD}$ in physics in 2007, both from the University Montpellier 2, Montpellier, France. Since 2008, he has been an assistant professor in the Laboratoire Charles Coulomb and Institut Universitaire de Technologie, University Montpellier 2. His research interests include vibrational and optical properties of pristine, functionalized, and individual carbon nanotubes, and the characterization of different materials by Raman spectroscopy.

Raul Arenal received his MS degree in materials science from the University of Joseph Fourier, Grenoble, France, in 2001, and he received his PhD in solid-state physics from Paris XI University, Orsay, France, in 2005. His research interest includes electron microscopy focused on materials science and nanoscience. He has published more than 100 articles/letters in international peerreviewed journals and around 50 peer-reviewed international conference proceedings.

Matthieu Paillet received his $\mathrm{PhD}$ in materials science from the University of Montpellier in 2005. Currently, he is holding a full-time CNRS researcher position at the Laboratoire Charles Coulomb, University of Montpellier. His research interests include carbon nanotubes and graphene physical properties.

Dmitry V. Rybkovskiy received his ME degree in nanotechnology in electronics from the Moscow State Institute of Radio Engineering, Electronics and Automation, Moscow, in 2010, and his $\mathrm{PhD}$ in condensed matter physics from A.M. Prokhorov General Physics Institute RAS, Moscow, in 2014. Currently, he is a postdoc at the Southern Federal University, Rostov-on-Don, Russia. His current research interests include theoretical investigation of layered semiconductors and carbon nanotubes, development, implementation, and use of electronic structure calculation methods.

Alexander V. Osadchy is a senior researcher of nanomaterials spectroscopy laboratory of A.M. Prokhorov General Physics Institute of Russian Academy of Sciences (GPI RAS). He received his PhD from GPI RAS in 2005. He received his master's degree with honor from M.V. Lomonosov Moscow State University in 2001. His current research interests include computer modeling and optical properties of carbon nanostructures, graphene, and other layered materials. $\mathrm{He}$ is the author of more than 17 articles.

Sergei B. Rochal is a professor of the first class at the Southern Federal University, Rostov-onDon, Russia. In 2002, he received the degree of a doctor of physical and mathematical sciences in condensed matter physics. He is the author of 55 journal papers and two books. His current research interests include self-assembly and properties of biological nano-objects and colloidal crystals, physical properties of carbon nanotubes, Landau theory of phase transitions in different systems, dynamics of crystals, lipid, and composite membranes.

Yury I. Yuzyuk received his MS degree in physics and his $\mathrm{PhD}$ in condensed matter physics both from the Rostov State University, Rostov-on-Don, Russia, in 1982 and 1989. He has 30 years of hands-on experience in Raman spectroscopy of molecular crystals, ferroelectric crystals, ceramics, thin films, superlattices, semiconductors, carbon nanotubes, and liquid crystals. He has coauthored more than 160 journal papers and three books. He participates in a number of research projects and is coordinator of two French-Russian projects.

Jean-Louis Sauvajol received his $\mathrm{PhD}$ in condensed matter physics from the University of Montpellier in 1977. From October 1977 to 1985, he was CNRS research associate at the Laboratoire de Dynamique des Cristaux Moléculaires of the University of Lille. He has 35 years of hands-on experience in Raman spectroscopy of molecular crystals, ODIC phases, conducting polymers, fullerenes, and carbon nanotubes. He has coauthored more than 230 journal papers. 